\title{
YNSA permanent acupuncture application for post-stroke syndrome
}

\author{
Molnar $\mathrm{I}^{1}$, Mate $\mathrm{A}^{1}$, Szoke $\mathrm{H}^{2}$ and Hegyi $\mathrm{G}^{2}$ \\ ${ }^{1}$ University of Pecs, Faculty of Health Sciences, Doctoral School of Health Sciences, Pecs, Hungary \\ ${ }^{2}$ Department of Complementary Medicine, Faculty of Health Sciences, University of Pecs, Pecs, Hungary
}

\begin{abstract}
Objectives: The aim of our newer study involving more numbers of patient was to investigate whether permanent acupuncture, especially Yamamoto's New Scalp Acupuncture (YNSA), and is of value in additional to standard post-stroke motor rehabilitation elevating QoL of patients with higher self-management.

Design: A prospective, assessor-blinded randomized control trial was carried out in an outpatient stroke rehabilitation unit with day hospital service again enrolled 520 cases. After inclusion, patients were stratified into control group and acupuncture group, randomly. All of stratified patients participated in gymnastic exercises and conservative pharmacological treatment according western rehabilitation protocol.
\end{abstract}

Outcome measures: The Barthel Index, the Rivermead Scale Index, the SP-36 questionnaire and the Visual Analogue Scale were used to follow the efficacy of treatment.

Results: In the acupuncture group, all the sensory, motor, and functional scores improved significantly during the examination period until 3 years after injury. The Barthel Index is increased from $4 \pm 2$ to $95 \pm 4$ in the acupuncture group. This index also increased in the control group (from $4 \pm 2$ to $75 \pm 4$ ), but the changes were significantly less than in the acupuncture group. A significant spontaneous recovery during the 3-year follow-up was found, but the YNSA treatment facilitated the functional recovery not only in moving but generally in cognitive functions and memory. Improved moving function and more flexible joints and ligaments were observed in comparison to the patients' condition prior to treatment.

Conclusions: The data suggest that the YNSA acupuncture is a useful complementer method to treat residual symptomes of stroke patients and enhance their quality of life, to achieve higher self-management level. As soon as possible after stroke this method should be started if cardio-pulmonal condition of patient is ballanced and allowed it. This study was a reproduction of our earlier research with more participants to allowed us to prove advantages of early application of permanent acupuncture treatment.

\begin{abstract}
Abbrevations: BI: Barthel Index; RMI: Rivermead Index; VAS: Visual Analogue Scale; YNSA: Yamamoto New Scalp Acupuncture; LTP: Long-term-potentiation; LU: Lung; LI: Large Intenstine; KI: Kidney; BL: Bladder; HT: Heart; SI:Small intestine; LR: Liver; GB: GallBladder; PC: Pericardium; TB: Triple Burner; SP: Spleen-Pancreas; ST:stomach
\end{abstract}

\section{Introduction}

Stroke is one of the leading causes of long-term adult disability, affecting approximately 120,000 people each year in the Hungary. The very word "stroke" indicates that no one is ever prepared for this sudden, often catastrophic event. Stroke survivors and their families can find workable solutions to most difficult situations by approaching every problem with patience, ingenuity, perseverance and creativity. Early recovery and rehabilitation can improve functions and sometimes remarkable recoveries for someone who suffered a stroke. Physical conditions post-stroke includes weakness, numbness and stiffness. After stroke, experiencing emotional changes can occur due to natural responses or changes caused by physical effects of the brain. Cognition problems: after stroke, the process of thinking, remembering and recognizing things called (cognition) can become challenging.

In our outpatient clinic we mainly see patients suffering from after-stroke symptoms, as muscle stiffness, hemiplegic limbs, motor (or rarely sensory) aphasia, depression of mood, epileptic seizures, difficulties in daily practice of life. This pathogenesis limits the ability to work, inhibits patients 'self-management, and significantly reduces their quality of life (QoL). Acupuncture has been used in Traditional Chinese Medicine to relieve pain and cure a variety of diseases for more than 2500 years. Acupuncture has been used for stroke in China and Korea for centuries, but scientific studies on this topic have only recently started to emerge. Some but not all of these trials have suggested a positive effect on recovery. There are numerous reports in the Chinese literature about the efficacy of acupuncture in stroke rehabilitation. However, the result is rarely quantitatively expressed by properly validated measures, and intention to treat analysis was never mentioned [1-3].

We introduced since 1989 an unique permanent acupuncture method for patients for avoid necessary weekly treatments and provide

Correspondence to: Hegyi Gabriella MD.PhD, Department of Complementary Medicine, Faculty of Health Sciences, University of Pecs, Pecs, Hungary, E-mail: drhegyi@hu.inter.net, gabika1947@gmail.com

Key words: stroke, post-stroke syndrome, permanent acupuncture, rehabilitation, quality of life (QoL), Visual Analogue Scale

Received: March 29, 2018; Accepted: April 12, 2018; Published: April 16, 2018 
them to achieve this additional rehabilitation form living far from clinic $[4,5]$

\section{Background/ Description of the investigated therapy}

Rehabilitation actually starts in the hospital as soon as possible after stroke. In patients who have stable cardiopulmonary situation, rehabilitation may begin within two days after the stroke has occurred and should be continued as necessary after release from the hospital. Depending on the severity of the stroke, rehabilitation options can include: A rehabilitation unit in the hospital with inpatient therapy

A subacute care unit controls

- A rehabilitation hospital with individualized inpatient therapy

- Home therapy

\section{Returning home with outpatient therapy}

- A long-term care facility that provides therapy and skilled nursing care

- YNSA (YAmamoto New Scalp Acupuncture System) is most frequently used for neuromusculoskeletal diseases, dysfunctions, and pain that may be either acute or chronic [6-8].

\section{Topographiai anatomia and neural connection}

YNSA acupuncture method is composed of tree categories: Basic points, Ypsilon points and Parietal Points. The Basic points are scalp acupuncture points used to treat pain and dysfunction of anatomical body parts that are directly related to the disease and dysfunctional process. Basic points are associated with small elevations or firmness in the subcutaneous tissue of the scalp at prescribed locations. YNSA also uses Ypsilon points, which relate to the 12 acupuncture meridians (LU, LI, KI, BL, HT, SI, LR, GB, PC, TB, SP, and ST). Since 1973, the YNSA method started to be applied to treat stroke patients worldwide; in our hospital, the method has been used since 1988, which is permanently developed by prof. Yamamoto $[9,10]$
Indications: neurological diseases, musculosceletal pains originated from different cause, muscle- , tendom stiffnes, rehabilitation of stroke, mood disorders

\section{Contraindications}

Instabil cardio-pulmonary situation

After acute heart attack stage

Cost-benefit ratio indicates YNSA usage as a complermentary valuable option to standard conservative therapy.

We applied YNSA and parietal points described first by $\mathrm{T}$. Yamamoto, (Figures 1 and 2)

\section{Objectives}

The aim of the investigation was, to assess the effects of YNSA on the circulation of the brain, the consecutive ischaemic pain, the changes in trophic disturbances and patient's quality of life - using validated objective and subjective measurements .

Our second aim was to observe possible complications and adverse reactions caused by the intervention, to compare our results with similar, previously published studies, to conclude on the applicability of this dry needling method on patients with post-stroke syndrome.

\section{Study design}

A prospective randomised case series was conducted in our outpatient rehabilitation-clinic over 36 months in Yamamoto Rehabilitation Institutre, Budapest, Hungary. Patients got detailed and clear explanation of the permanent acupuncture intervention and the preceeding tests necessary for the study and signed written informed consent.The study was following the principles of the Declaration of Helsinki, with the written approval of the Local Research Ethics Committee (under registration number: 257/2016).

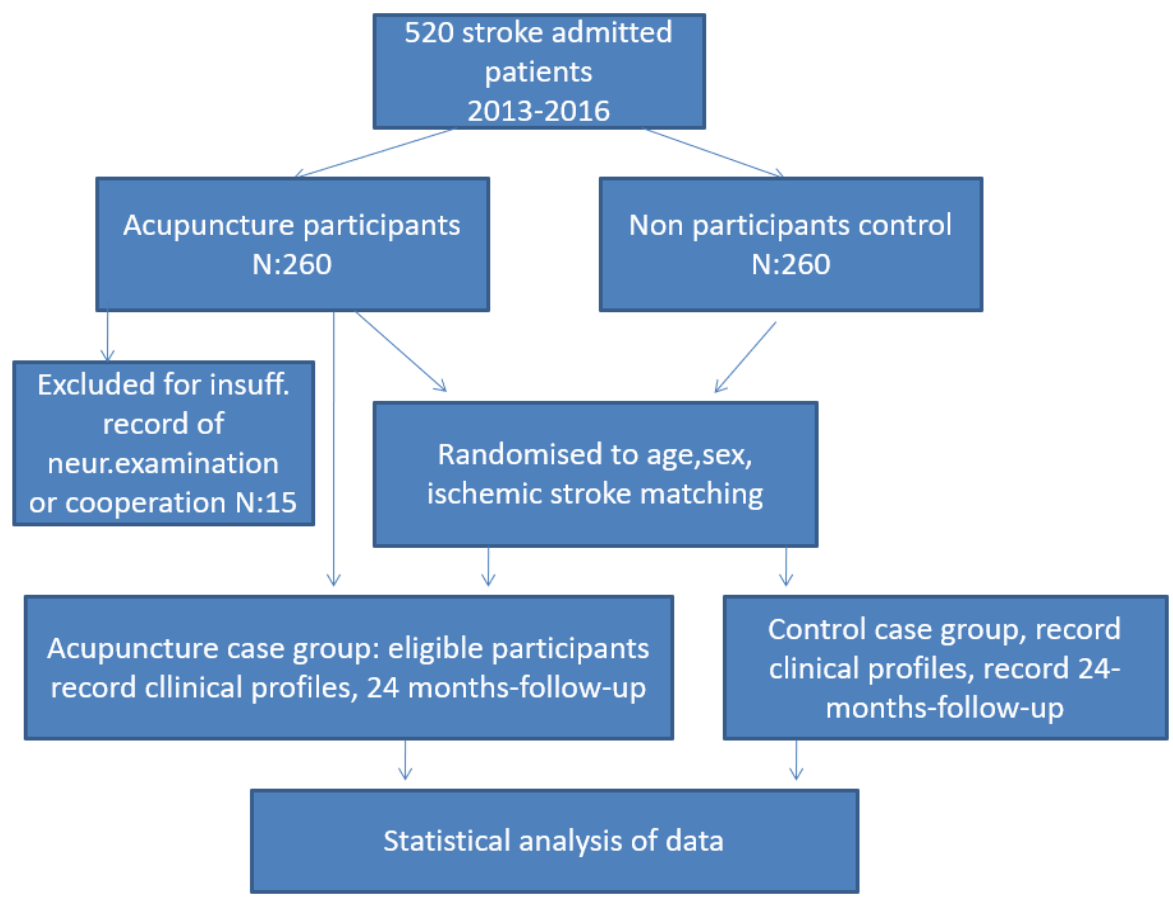

Figure 1. Treatment of 260 patients with YNSA and the other 260 subjects were the nontreated control group. 


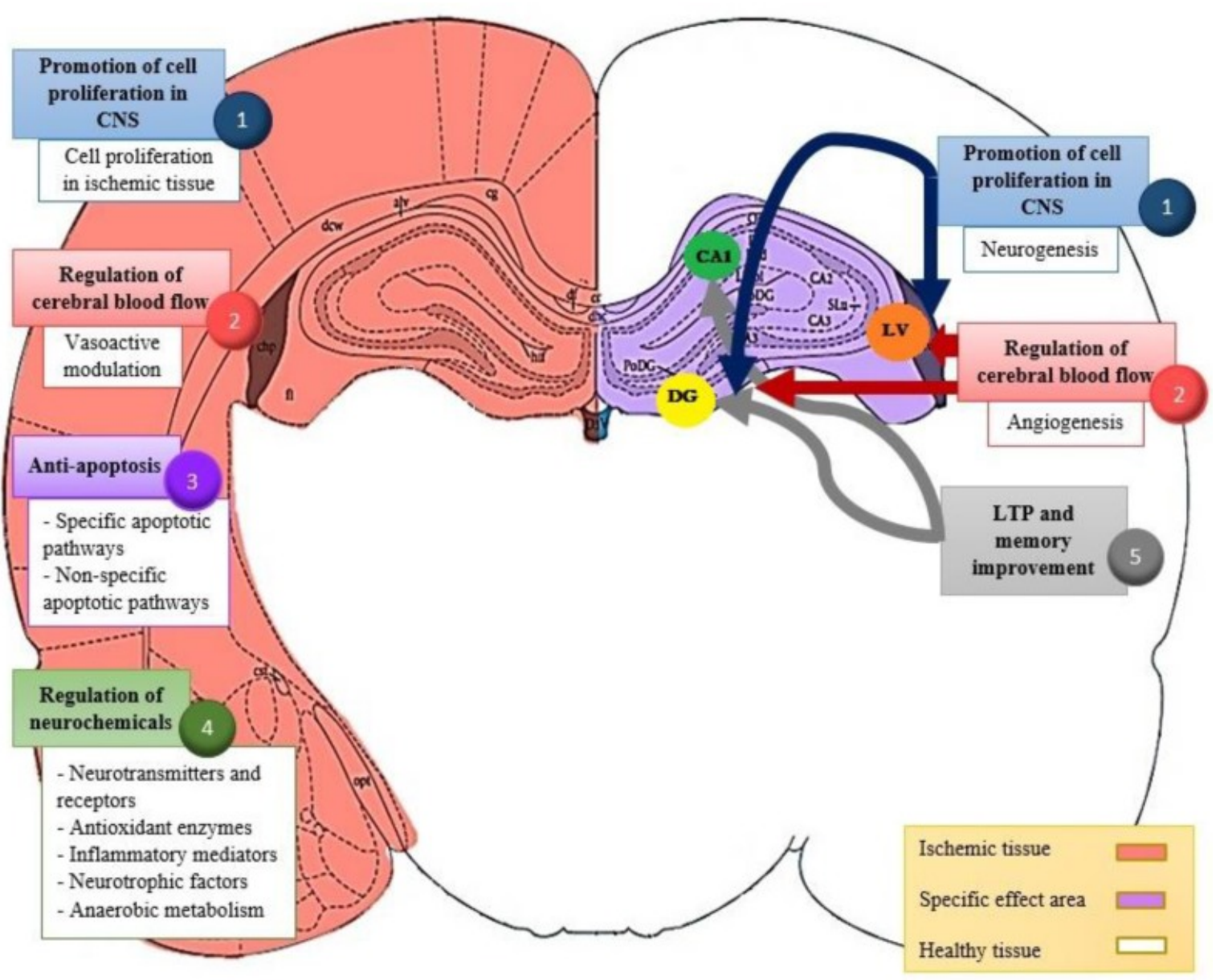

Figure 2. Demaged and healthy functions of CNS (after Lynch MA)

\section{Material -Patients}

Selection of patients: Under real life conditions all self selected patients were included, if they fulfilled the inclusion criteria and singned their informed consent.

\section{Inclusion crieteria}

- stroke in past 2 years

- hemiparalyse, stiffness of flector muscles of hand and foot persisted,

- age $18-78$ years,

- clear consciousness,

- balanced cardio-pulmonal state

Exclusion criteria: imbalance of cardio-respiratory system

\section{Outcomes and Method}

Objective outcomes:1) Measurement Barthel index and Rivermead scale, before treatrment and on day 30, 60, 90, 120,360 [11-13].

Subjective outcomes: -Self-evaluation of the patients

1) VAS [14] was used for defining the intensity of pain,movement, modd disorders, QoL

Scale 0-10:
9 to $10=$ no pain, better movement, minimal stiffness

7to $8=$ mild pain, mild stiffness, barely affecting everyday life;

4 to $6=$ moderate pain: significantly bothers everyday life, mild depression

1 to $3=$ severe pain: paralyse, hinders everyday life, deep depression

2) SF-36 questionnaire for monitoring changes in quality of life [15-19].

Clinical setting/ treatment: Diagnostics were performed before entering the study: typical complains and symptoms of the disease. Instrumental investigations such as MRI, CT were performed during the previous conventional treatment. Patients continued to take other previously given adjusted medications and gymnastic exercises since they had no influence on our monitoring.

\section{Steps of the investigation}

1) Anamnesis

2) Physical examination

3) Measurements before the intervention (Barthel index, Rivermead index, VAS, SF 36 questionnaire)

4) Intervention: YNSA and parietal points as a permanent form of acupuncture. 
5) Measurements after the intervention

6) We observed every patient for about $1 / 2$ hour after the intervention. As their vital parameters were stable, they were allowed to leave our ambulance.

7) The intervention was repeated once a month, that is six times altogether.

\section{Method}

Recruitment commenced between 2013 and 2017, after 520 patients had been enrolled ( 260 to receive acupuncture treatment and 260 for control). 260 (50\%) consecutive patients admitted to the stroke rehabilitation unit were included in the study after informed consent. Inclusion criteria were the following: (1) patients with hemorrhagic or ischemic stroke,(2) admission within 6 weeks of stroke, (3) patients should have movement coordination problems including muscular atrophy, central paralysis, and also myasthenic symptoms, and (4) the degree of brain damage of stroke patients did not exceed $30 \%$ of damaged brain tissue. The last statement is important, because when the brain damage exceeds $30 \%$, usually the dry needling treatment would not be successful [20-23]. The size and anatomical location of stroke lesions is evaluated by magnetic resonance imaging using subtraction lesion analysis. Exclusion criteria were the following: (1) no motor deficit, (2) hemodynamic instability, (3) history of dementia, and (4) inability to give consent because of impaired cognition or receptive aphasia. The authors treated 260 patients with YNSA and the other 260 subjects were the nontreated control group (Figure 1). All patients gave informed consent to participate in the study, which was performed according to the guidelines of the local ethics committee. The participants were not informed of the possibility of being assigned to either acupuncture or no acupuncture group. All the recruited patients went under rehabilitation program using the Hungarian standard rehabilitation protocol. 260 of the patient received additional acupuncture therapy using the dry needling method, and these patients were regarded as the acupuncture group. The other 260 patients without acupuncture therapy were regarded as the control group, and they only received necessary rehabilitation therapies [24]. The average age was $58.6-10.4$ and 59.8 - 9.6 years (in the acupuncture and control group, respectively). In both groups, 92 patients had ischemic and 92 had hemorrhagic stroke [Table 1].

\section{Steps of the intervention:}

- Patient lying with slightly outstretched neck

- With the finger we palpate the small areas on scull to find any softer/ harder area which corresponds YNSA points (according to YNSA map, Figure 2.3)

- Over the lying finger a fine 3-4 cm long needle (size 12) is pierced dorsally through the skin. After getting the sensitive area contact we push the mandrine towards, so the absorbent monofilament goes

Table 1. Demographic and Clinival Characteristics of treated patients by Group.

\begin{tabular}{|c|c|c|}
\hline Male gender, $\%$ & $\mathbf{1 4 5}$ & $\mathbf{1 1 5}$ \\
\hline Mean age in years (SD) & $59.8(9.6)$ & $58.6(10.4)$ \\
\hline Current smoking, \% & 86 & 60 \\
\hline High school or higher educational level, \% & 90 & 56 \\
\hline Depression (clinical diagnosis), \% & 25 & 24 \\
\hline Very intense pain at presentation, \% & 50 & 45 \\
\hline Lesion of brain tissue, \% & 15.8 & 17.1 \\
\hline Ischemic stroke vs all, \% & 92 & 92 \\
\hline
\end{tabular}

to tissue. After withdrawing the needles with mandrine we save the insertion.

- as a result: a few minutes later can be seen the effect, as improved movement ability, which lasts $2-3$ weeks.

- The next intervention follows the same procedure

Traditionale acupuncture therapy (dry needling) is usually done by the insertion of thin metal needles (Gauge 30) to the acupoints, and this is followed by slow manual twisting action of the acupuncturist. The needles, once correctly inserted into the acupoints, can also be stimulated electrically. The application of needles might even be a noxious stimulus causing autonomic hyperreflexia. Another, later developed acupuncture method is the YNSA, where we applied small absorbable threads can make a continuous biostimulation of acupoints. The treatment period took 12 months, by monthly one session.

We compared the result before and after the treatment. The last follow up was performed on day 365 to detect - not just temporary but - durable effects of the intervention.

Statistical evaluation:Change in pain intensity was calculated by the $\chi^{2}$ test. Changes before and after treatment in each dimension of the SF-36 questionnaire, were performed by Wilcoxon's test. Data were recorded and analyzed using the SYSTAT 10 program package, according to previous studies and the SF-36 manual. The correlation-calculation method and the 'Excel and built-in functions' software package were used for data analysis.Level of $\mathrm{p}<0.05$ was determined as significant.

\section{Results}

\section{Regarding baseline characteristics}

Data of 520 examined and 260 treated patients were collected. All other patients seen in this time in the outpatient pain-clinic with same symptoms did not fulfill the inclusion criteria or were not willing to participate in the study. Distribution of the investigated patients was: 145 male (mean age 59.0 of years) and 115 female (mean age 41.2 of years) patients were detected (Table 1).

The primary outcome was the change in Barthel ADL score. Secondary outcome measures included National Institutes of Health Stroke Scale score, motoricity index, quality of life (EQ-5D [EuroQoL-5 Dimensional form] and EQ-VAS [EuroQoL-Visual Analog Scale]),

\section{Regarding outcomes}

The average follow up time was 2 years after treatment.

15 drop outs were detected (Figure 1).

\section{Objective outcomes}

1) Aggregated changes in the VAS and QoL showed significant improvement $(\mathrm{p}=0.002)$. (Figure 2$)$.

2) Aggregated changes in the parameters showed significant improvement $(\mathrm{p}<0.05)$.

in Barthel index, in Rivermead Scale (Figure 2).

\section{Subjective outcomes}

1) VAS values: 49 patients had pain value of 9 , and 54 patients had pain value of 7 before treatment. After the treatment 12 patients marked value 5,18 patients marked pain value 2 , and 14 patients marked pain value 0 (Figure 3 ) So changes in VAS values showed a significant improvement $(\mathrm{p}=0.001)$. 
2) In the dimensions of the quality of life the aggregated change of SF-36 questionnaire also showed a significant improvement $(\mathrm{p}=0.005)$ (Tables 2 and 3).

There was no relevant difference between male and female patients concerning the dimension of changes in the averaged values, but the age paid relative big role in hope and imagination of the future of involved patients.Patients imaginations with younger age were more positive as older's.

\section{Adverse reactions}

No lasting complications were detected in any case.

\section{Discussion}

Several previous stroke disease-specific studies confirm, that additional acupuncture teratment of post stroke patents is an unsuccessful treatment. We aimed to compare our results with the outcomes of other similar studies lately published where the outcome of treatment was elevate the movement of patients and QoL $[25,26]$. However, we could not find any case reports that aimed to improve circulation of the limbs in patients with post-stroke syndrome by treating with dry needling, and that followed up changes in quality of life .

According to ethical and professional reasons we create a control group for patients with same problems. Bias were taken into account and minimized.

The aim of this study was to examine the long-term effects of repeated embedded YNSA treatment on balance and activities of daily living in postacute stroke patients compared with the noacupuncture group. "So, what is the truth about acupuncture in stroke rehabilitation?" was asked at the end of the Introduction section. In the authors' opinion at least two things are important to determine the
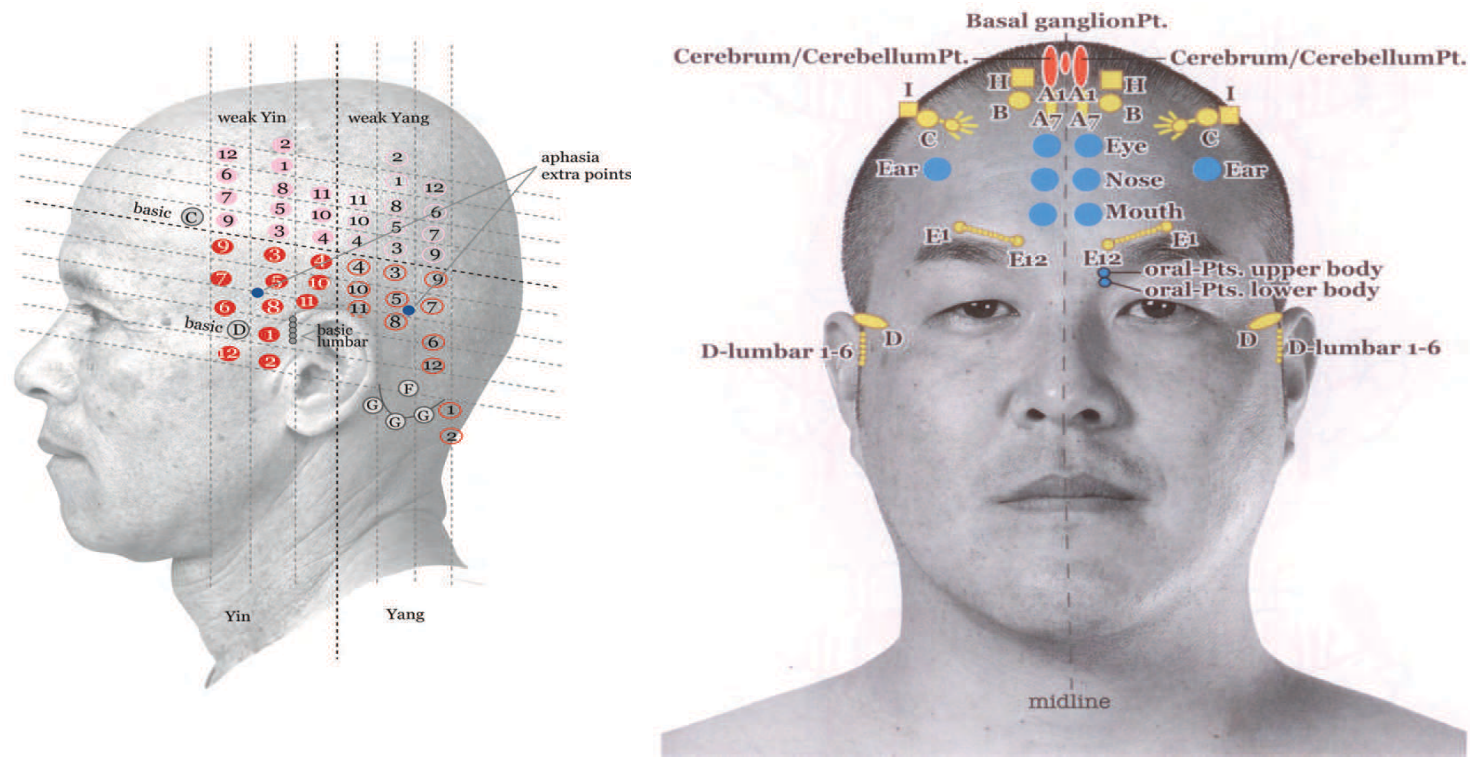

Figure 3. YNSA MAP, Ypsilon and Basic points on scalp

Table 2. Pretreatment and posttreatment scores of the SF-36 questionnaire ( $1=$ before therapy, $2=$ after therapy; $*$ indicates significant difference)

$\mathrm{FP}=$ Physical functioning, Physical health Problems; RP = Physical Role functioning, $\mathrm{BP}=$ Bodily pain; $\mathrm{GH}=$ General health perceptions; $\mathrm{VT}=\mathrm{Vitality}$, energy/fatique; SF $=$ Social role Functioning; RE = Emotional Role functioning; Emotional health problems; $\mathrm{MH}=$ Mental Health, Emotional well-being.

\begin{tabular}{|c|c|c|c|c|c|c|c|}
\hline $\begin{array}{c}\text { SF-36 } \\
\text { dimensions }\end{array}$ & Number of cases & $\begin{array}{l}\text { Two-sided } \\
\text { probability }\end{array}$ & Median & Mean & Standard deviation & 25th percentile & 75th percentile \\
\hline FP-1 & \multirow{2}{*}{245} & \multirow{2}{*}{$\mathrm{p}=0.005^{*}$} & 72.500 & 67.917 & 17.896 & 57.5 & 85 \\
\hline FP-2 & & & 90.000 & 86.250 & 17.854 & 83.75 & 96.25 \\
\hline RP-1 & \multirow{2}{*}{245} & \multirow{2}{*}{$\mathrm{p}=0.007^{*}$} & 25.000 & 29.167 & 33.428 & 0 & 0 \\
\hline RP-2 & & & 100.000 & 79.167 & 39.648 & 87.5 & 100 \\
\hline BP-1 & \multirow{2}{*}{245} & \multirow{2}{*}{$\mathrm{p}=0.002 *$} & 26.500 & 23.750 & 13.897 & 16 & 34 \\
\hline BP-2 & & & 70.000 & 69.750 & 14.759 & 62 & 83 \\
\hline GH-1 & \multirow{2}{*}{245} & \multirow{2}{*}{$\mathrm{p}=0.011 *$} & 22.500 & 22.917 & 18.148 & 8.75 & 31.25 \\
\hline GH-2 & & & 26.000 & 28.083 & 19.033 & 15.25 & 37 \\
\hline VT-1 & \multirow{2}{*}{245} & \multirow{2}{*}{$\mathrm{p}=0.011^{*}$} & 42.500 & 41.250 & 20.794 & 30 & 51.25 \\
\hline VT-2 & & & 57.500 & 55.833 & 22.139 & 48.75 & 68.75 \\
\hline SF-1 & \multirow{2}{*}{245} & \multirow{2}{*}{$\mathrm{p}=0.004 *$} & 50.000 & 40.583 & 25.681 & 25 & 50 \\
\hline SF-2 & & & 75.000 & 80.250 & 18.061 & 75 & 100 \\
\hline RE-1 & \multirow{2}{*}{245} & \multirow{2}{*}{$\mathrm{p}=0.038^{*}$} & 16.500 & 38.833 & 46.789 & 0 & 100 \\
\hline RE-2 & & & 100.000 & 75.000 & 45.227 & 75 & 100 \\
\hline MH-1 & \multirow{2}{*}{245} & \multirow{2}{*}{$\mathrm{p}=0.005^{*}$} & 50.000 & 48.667 & 17.042 & 39 & 60 \\
\hline MH-2 & & & 60.000 & 60.667 & 15.144 & 51 & 73 \\
\hline
\end{tabular}


-Treated-group, $\square$-Control-group
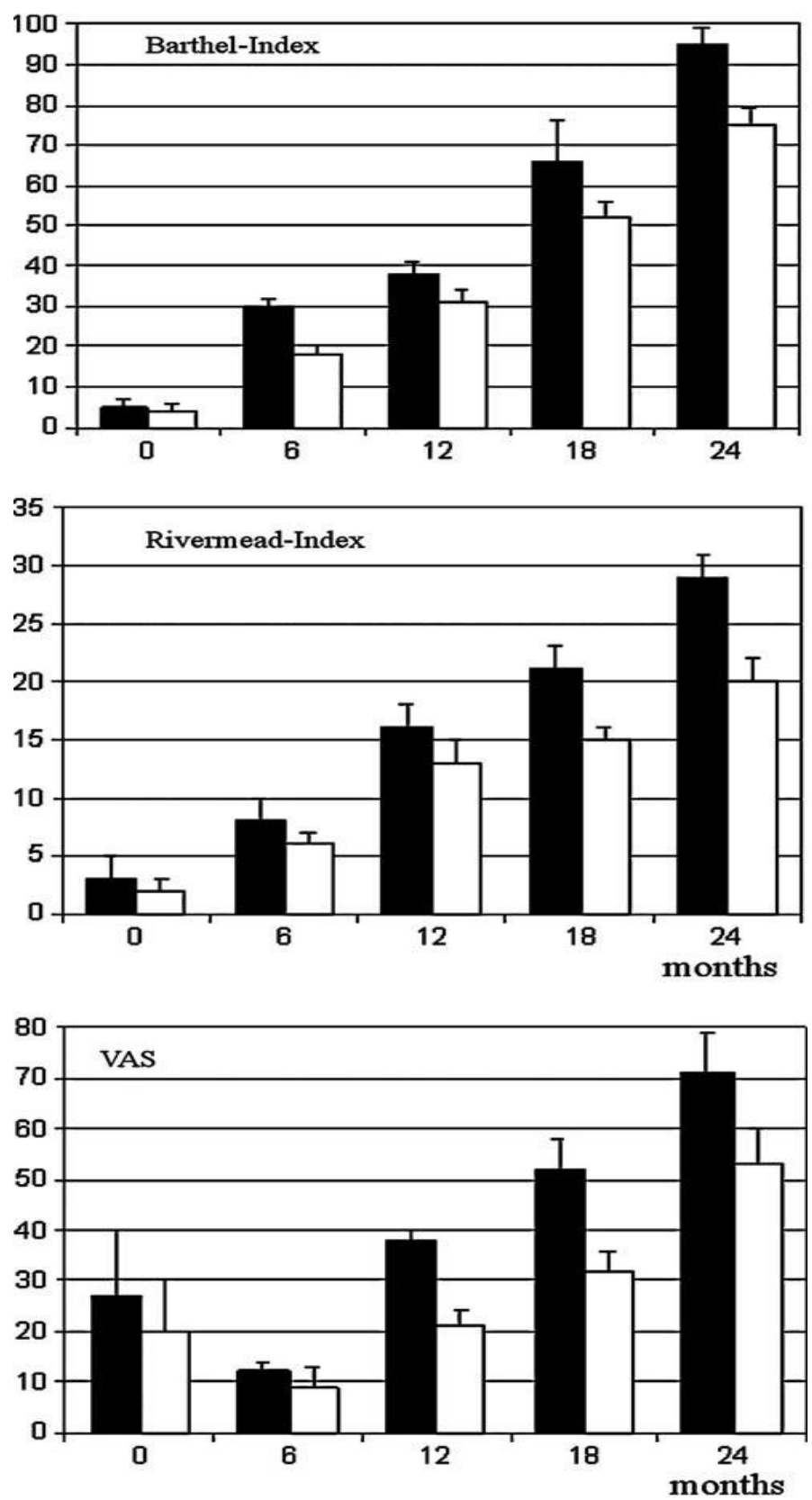

Table 3. Changes of Barthel Index, Rivermead Index, and Visual Analogue Scale (VAS) in acupuncture (black columns) and control (white columns) group.

efficacy of the acupuncture treatment in stroke rehabilitation. The most important is to enhance the life quality of the stroke patients during and after the acupuncture treatment. The second thing is to find the possible link between the acupuncture treatment and the cellular mechanisms that activated in the recovery period of stroke. Previous studies have reported the ineffectiveness of sensory stimulation by acupuncture or transcutaneous electrical nerve stimulation, functional electrical stimulation, electro- myographic feedback, force feedback or body weight sup- ported treadmill training on balance in patients with stroke [25]. Recent evidences have shown that impairment-focused programs (biofeedback, electric stimulation, muscle strengthening) failed to generate functional improvements whereas therapies that administered functional training improved activity levels [26]. Unfortunately, there are only a few previous data published about the YNSA treatment in stroke survivors. However, in the current study, the generally used BI, the RMI, SP-36 and the VAS were applied to determine the changes of life qualities of participants. The patients were followed in a 2-year period after the stroke. All of the four parameters were increased in the 24-month follow-up period in the acupuncture and control group; however, the changes were significantly greater in the acupuncture group than in the control group. The data suggest that the YNSA is a useful method to treat the stroke patients and enhance the quality of life. Using BI, RMI, SP-36 and VAS for the motor-status evaluation, the results showed that YNSA is effective in patients with stroke (ischemic and hemorrhagic) for motor recovery. To the authors' knowledge, this is the reproduction of our first study in this topic, to demonstrate that YNSA has beneficial effects on body motor performance in patients with stroke [27]. In terms of functional improvement, both the study group and the control group showed im- provement in functional performance at follow-up assessments. However, there was a tendency for the study group to be superior to the control group in the total scores at follow-up stages, and at the end of the follow-up period it did reach statistical significance. However, the link still must be found between the acupuncture and the cellular changes after stroke. Some evidence suggests that the acupuncture stimulation with needle has some cellular and tissue effects and that the acupuncture can enhance the release of transmitters that can facilitate angio- and neurogenesis in the central neural system [28]. On a cellular level, the phenomenology of neural repair after stroke has been defined and unique regenerative environments in the poststroke brain identified. These data suggest that acupuncture facilitates some molecular and cellular mechanisms that have an important role in the angiogenesis, neurogenesis, and tonic neuronal inhibition in brain tissue [29-33]. This it can be concluded that acupuncture, especially the YNSA, is a useful complementary tool to facilitate stroke rehabilitation.

\section{Conclusion}

Hemiparalyse, depression and chronic pain causes a decline of the quality of life. Although analgesia is obviously a symptomatic therapy, our results showed, that - independently from the underlying cause - the permenent technique of acupuncture specialized to YNSA and scalp points is able to achieve a durable improvement in symptoms and quality of life of patients.By treating the sclap acupuncture points, circulation and innervation of the hands and legs can be improved significantly is already proved [30-32]. Does acupuncture really work to help stroke victims improve? Many studies involving thousands of patients have been published in China and Japan, and 2 of 3 studies from Scandinavia, demonstrated significant help. These studies indicate that patients get well faster, perform better in self-care, require less nursing and rehabilitation therapy, and use less healthcare dollars. However, since most studies come from China, they get little credence from the Western medical community because researchers in China do not appear to be published unless their results are highly positive, so publication bias is possible. Overall permanent acupuncture applying YNSA presents an added value in the complex treatment of patients with post-stroke syndrome.

From the available evidence, acupuncture may have beneficial effects on improving dependency, global neurological deficiency, and some specific neurological impairments for people with stroke in the convalescent stage, with no obvious serious adverse events [34-38] (Figure 4)

Further investigations can be done with larger number and longer follow-up of patients. These data suggest that the use of YNSA therapy 


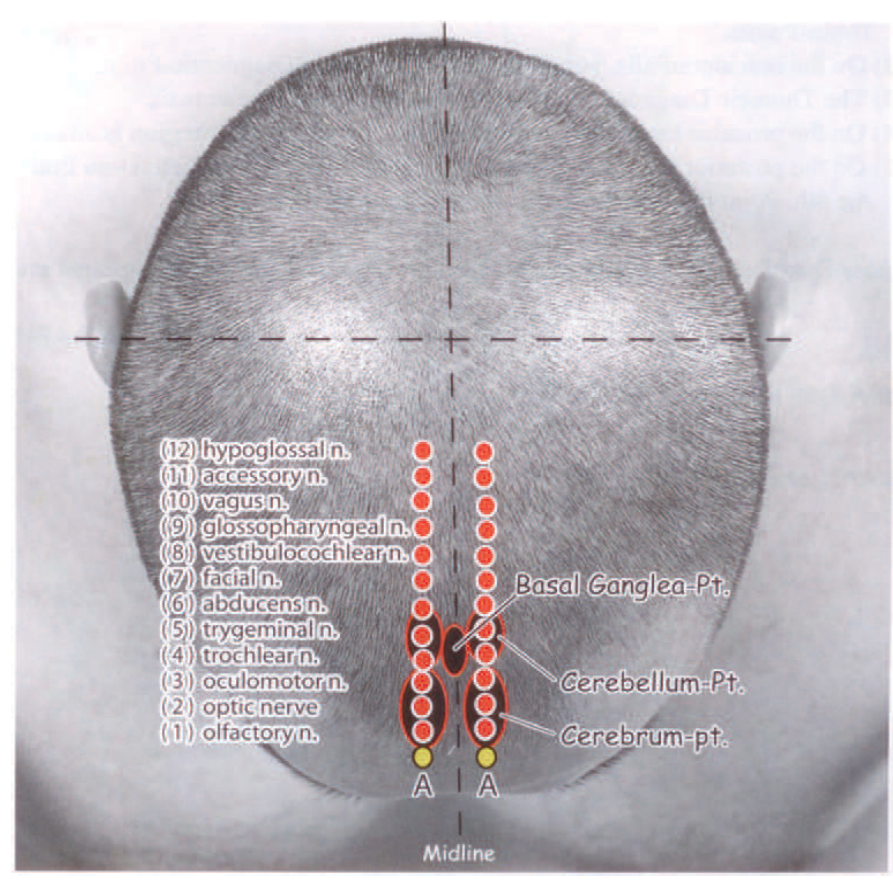

Figure 4. YNSA MAP, parietal points for nerves

with embedded threads after stroke can contribute to significant neurologic and functional recoveries.

We proved higher positive effect in patients with smaller demaged brain area, (under 30\%) earlier starting the reatment after Stroke and younger age.

There are several limitations of this study: Because there was no sham control, it is not known how much the YNSA intervention contributed to the improvement of subjects in the acupuncture group be- cause it is not known how much of the improvement was due to placebo effect. Further data exploration suggested that these complete case analyses are likely to provide valid statistical interferences.

\section{Acknowledgements}

First of all many grateful thanks to prof. Yamamoto to teach us this system. Acupuncture according to YNSA, belongs to the CAM methods in more European countries [39] (CAMbrella Pan-european project, 2010-2012), is one of it's advanced methods too. In some countries (Austria, Germany, Hungary, Czech R, Slovakia, Greek) it is accepted official CAM methods for medical doctors only, providing better QoL of patient for different diseases which are involved in it's indication. Pecs University CAM Department has organized more official courses for MDs according to Yamamoto New Scalp Acupuncture.

\section{Conflicts of interest}

The authors declare no conflict of interest in this study.

\section{References}

1. Ernst E (2004) The need for scientific rigor in studies of complementary and alternative medicine. Am J Public Health 94: 1074- 1075

2. Lina MC, Shiang SH, MacDonald I, Geng LJ, Lee YC, Chen YH, et al. (2017) Mechanisms of Acupuncture Therapy in Ischemic Stroke Rehabilitation: A Literature Review of Basic Studies. Int J Mol Sci 8: 2270

3. Li X, Wang Q (2013) Acupuncture therapy for stroke patients. Int Rev. Neurobiol 111: 159-179.
4. Hegyi G, Szigeti G (2012) Rehabilitation of stroke patients using Yamamoto New Scalp Acupuncture: a pilot study. J Altern Complement Med 18: 971-977

5. Hegyi G, Pfeiffenroth A, Csutortoki K (2012) Hungarian Military Use of Acupuncture: Recent Clinical Trials and Accumulated Survey Data. Medical Acupuncture 24: 152-155.

6. Schockert T, Schnitker R, Boroojerdi B (2009) Cortical activation by Yamamoto new scalp acupuncture (YNSA) in the treatment of stroke patients-a sham-controlled Study aided by functional magnetic resonance imaging (fMRI) Dt. Zeitschr f Akup 1: 21-29.

7. Schockert T, Beissner F (2010) Neurophysiological Correlates of the Effect of YNSA for Patients with Chronic Pain of the Locomotor System - Basic YNSA Research by Means of PET-CT. Deutsch Ztschr f Akup 2: 8-13

8. Schockert T, Schnitker R, Boroojerdi B, Vietzke K, Qua Smith I, Yamamoto T, et al. (2010)

9. Cortical Activation by Yamamoto New Scalp Acupuncture (YNSA) in the Treatment of Stroke Patients - A Sham-controlled Study Using Functional Magnetic Resonance Imaging (fMRI). Acupunct Med 4: 212-214

10. Yamamoto T, Yamamoto H, Yamamoto MM (2005) Yamamoto Neue Scha"delakupunktur. Bad Ko" tzting: VGM AU: 9-16

11. Schockert T (2007) YNSA: Individual therapie durch Halsdiagnos- tik. Komplement Integr Med 10: 1-8

12. Mahoney FI, Barthel DW (1965) Functional evaluation: The Barthel Index. Md State Med J 14: 61-65.

13. Granger CV, Dewis LS, Peters NC (1979) Stroke rehabilita- tion: Analysis of repeated Barthel index measures. Arch Phys Med Rehabil 60: 14-17.

14. Wade DT, Collin C (1988) The Barthel ADL Index: A standard measure of physical disability? Int Disabil Stud 10: 64-67.

15. Ahlsioo B, Britton M, Murray V, Theorell T (1984) VAS disablement and quality of life after stroke. Stroke 15: 886-890.

16. Hawker GA, Mian S, Kendzerska T, French M (2011) Measures of adult pain: Visua Analog Scale for Pain (VAS Pain), Numeric Rating Scale for Pain (NRS Pain), McGill Pain Questionnaire (MPQ), Short-Form McGill Pain Questionnaire (SF-MPQ), Chronic Pain Grade Scale (CPGS), Short Form-36 Bodily Pain Scale (SF-36 BPS), and Measure of Intermittent and Constant Osteoarthritis Pain (ICOAP). Arthritis Care Res 11: S240-S252

17. Bunevicius A (2017) Reliability and validity of the SF-36 Health Survey Questionnaire in patients with brain tumors: a cross-sectional study. Health Qual Life Outcomes 15: 92.

18. Salim S, Yamin M, Alwi I, Setiati S (2017) Validity and Reliability of the Indonesian Version of SF-36 Quality of Life Questionnaire on Patients with Permanent Pacemakers. Acta Med Indones 49: 10-16.

19. Ware J (2000) SF-36 Health Survey Update. Spine 25: 3130-3139. [Crossref]

20. Bunevicius A (2000) Reliability and validity of the SF-36 Health Survey Questionnaire in patients with brain tumors: a cross-sectional study. Health Qual Life Outcomes 15 : 1-92. [Crossref]

21. van Peppen RPS, Kwakel G, Wood-Dauphinee S (2004) The impact of physical therapy on functional outcome after stroke: What's the evidence? Clin Rehabil 18: 833-862.

22. Alexander LD, Black SE, Gao F (2010) Correlating lesion size and location to deficit after ischemic stroke: the influence of accounting for altered peri-necrotic tissue and incidental si- lent infarcts. Behav Brain Funct 6: 6-15

23. Endres M, Nyary I, Ba'nhidi M, Dea'k G (1990) RMI stroke rehabilitation: A method and evaluation. Int $J$ Rehabil Res 13: 225-236.

24. Park J, Hopwood V, White AR, Ernst E (2001) Effectiveness of acupuncture for stroke: A systematic review. J Neurol 248: 558-563.

25. Sze FK, Wong E, Or KKH (2002) Does acupuncture improve motor recovery after stroke? A meta-analysis of randomized controlled trials. Stroke 33: 2604-2619.

26. Lijun Bai, Yin Tao, Dan Wang, Jing Wang, Chuanzhu Sun, Nongxiao Hao, et al. (2014) Acupuncture Induces Time-Dependent Remodelling Brain Network on the Stable Somatosensory First-Ever Stroke Patients: Combining Diffusion Tensor and Functional MR Imaging. Evidence-Based Complementary and Alternative Medicine 1: 1-7

27. Seong-Uk Park, Ae-Sook Shin, Geon-Ho Jahng, Sang-Kwan Moon, Jung-Mi Park (2009) Effects of Scalp Acupuncture Versus Upper and Lower Limb Acupuncture on Signal Activation of Blood Oxygen Level Dependent (BOLD) fMRI of the Brain and Somatosensory Cortex. The Journal of Alternative and Complementary Medicine 15: $1193-1200$ 
28. Lynch MA (2004) Long-term potentiating and memory. Physiol Rev 84: 87-136.

29. Li X, Wang Q (2013) Acupuncture therapy for stroke patients. Int Rev. Neurobiol 111: 159-179.

30. Carmichael ST (2008) Themes and strategies for studying the biology of stroke recovery in the poststroke epoch. Stroke 39: 1380-1388.

31. Ding Y, Yan Q, Ruan JW (2009) Electro-acupuncture pro- motes survival, differentiation of the bone marrow mesen- chymal stem cells as well as functional recovery in the spinal cord-transected rats. BMC Neurosci 10: 35.

32. DMF C, Mons N, Karpan JW (1995) Adenylyl cyclases and the interaction between calcium and cAMP signaling. Nature 374: 421-424.
33. Ghosh A, Carnahan J, Greenberg ME (1994) Requirement for BDNF in activitydependent survival of cortical neurons. Science 263: 1618-1623.

34. Hansen MR, Zha XM, Bok J, Green SH (2001) Multiple distinct signal pathways, including an autocrine neurotrophic mechanism, contribute to the survival-promoting effect of depolarization on spiral ganglion neurons in vitro. $J$ Neurosci 21: 2256-2267.

35. Fu LW, Longhurst JC (2009) Electroacupuncture modulates vlPAG release of GABA through presynaptic cannabinoid CB1 re- ceptors. J Appl Physiol 106: 1800-1809.

36. CAMbrella Paneuropean Project, -www.cambrella.eu (https://cambrella.eu/home php?il $=8 \& \mathrm{l}=\mathrm{deu})$.

Copyright: $(\mathbb{O} 2018$ Molnar I. This is an open-access article distributed under the terms of the Creative Commons Attribution License, which permits unrestricted use, distribution, and reproduction in any medium, provided the original author and source are credited. 